Open Access

\title{
Parallel simulations for QUAntifying RElaxation magnetic resonance constants (SQUAREMR): an example towards accurate MOLLI T1 measurements
}

\author{
Christos G. Xanthis ${ }^{1,2}$, Sebastian Bidhult ${ }^{1}$, George Kantasis ${ }^{3}$, Einar Heiberg ${ }^{1,4,5}$, Håkan Arheden ${ }^{1}$
} and Anthony H. Aletras ${ }^{1,3^{*}}$

\begin{abstract}
Background: $\mathrm{T1}$ mapping is widely used today in CMR, however, it underestimates true $\mathrm{T1}$ values and its measurement error is influenced by several acquisition parameters. The purpose of this study was the extraction of accurate T1 data through the utilization of comprehensive, parallel Simulations for QUAntifying RElaxation Magnetic Resonance constants (SQUAREMR) of the MOLLI pulse sequence on a large population of spins with physiologically relevant tissue relaxation constants.

Methods: A CMR protocol consisting of different MOLLI schemes was performed on phantoms and healthy human volunteers. For every MOLLI experiment, the identical pulse sequence was simulated for a large range of physiological combinations of relaxation constants, resulting in a database of all possible outcomes. The unknown relaxation constants were then determined by finding the simulated signals in the database that produced the least squared difference to the measured signal intensities.

Results: SQUAREMR demonstrated improvement of accuracy in phantom studies and consistent mean T1 values and consistent variance across the different MOLLI schemes in humans. This was true even for tissues with long T1s and MOLLI schemes with no pause between modified-Look-Locker experiments.

Conclusions: SQUAREMR enables quantification of $\mathrm{T} 1$ data obtained by existing clinical pulse sequences. SQUAREMR allows for correction of quantitative CMR data that have already been acquired whereas it is expected that SQUAREMR may improve data consistency and advance quantitative MR across imaging centers, vendors and experimental configurations. While this study is focused on a MOLLI-based T1-mapping technique, it could however be extended in other types of quantitative MRI throughout the body.
\end{abstract}

Keywords: Magnetic resonance imaging, Mapping, MOLLI, Simulations, Relaxometry

\section{Background}

In the field of cardiovascular magnetic resonance (CMR) T1 mapping, quantitative measures of myocardial and blood T1 enabled the calculation of important myocardial biomarkers, such as extracellular volume (ECV) fraction and native $\mathrm{T} 1$ in the myocardium $[1,2]$. Recent

\footnotetext{
*Correspondence: aletras@hotmail.com

'Cardiac MR Group, Department of Clinical Physiology and Nuclear Medicine, Skåne University Hospital Lund, Lund University, Lund, Sweden

${ }^{3}$ Laboratory of Computing and Medical Informatics, School of Medicine, Faculty of Health Sciences, Aristotle University of Thessaloniki, Thessaloniki, Greece Full list of author information is available at the end of the article
}

advances in T1 mapping include various new techniques such as Modified Look-Locker inversion recovery (MOLLI), Saturation recovery single-shot acquisition (SASHA), AIR, SAPPHIRE and Shortened MOLLI (ShMOLLI) [3-7]. Moreover, there have been significant efforts towards understanding how accuracy, precision and reproducibility of these methods are affected by acquisition and post-processing parameters [8-11].

While T1 mapping has the potential to improve patient diagnosis, the challenge remains in acquiring reliable data in terms of accuracy and precision. 
Different T1 mapping methods and parameter sets should yield similar results for a specific tissue type. However, the complex nature of the underlying physics involved and the multitude of parameters that affect image acquisition and post-processing do not allow for consistent reference $\mathrm{T} 1$ values of normal myocardium and blood across all methods. Examples of this inconsistency include recent studies on cardiac T1 mapping, which have reported different ranges of T1 values for normal myocardium and blood depending on the methods being used [3, 6, 11-13].

More recently, a single vendor, multicenter clinical study [14] demonstrated reproducibility of myocardial T1 values and provided data from healthy myocardium. Interestingly, the reported $\mathrm{T} 1$ values in the literature covers a large range depending on the methods [8]. The T1 mapping technique (MOLLI) and the acquisition parameter set used in the aforementioned multicenter study are listed [14] as limiting factors in terms of accuracy when compared to other setups (different T1 mapping techniques, acquisition scheme, flip angle etc.) $[8,10]$.

The development of new CMR techniques for obtaining quantitative information usually involves a two-step process: acquisition of images from the MRI scanner with a custom designed pulse sequence and postprocessing of the acquired images through a data fitting procedure with closed form equations. Recently, a novel approach to collect and process images for extracting quantitative data from MRI experiments was proposed [15]. This new approach relies on making pseudorandom measurements and comparing the rapidly acquired data against a large dictionary of Bloch simulations. When the matching dictionary entry is found then the tissue constants are known since each of the entries was created based on specific tissue constants. This approach is new in quantitative MRI but requires a uniquely designed pulse sequence with a continuous variation of the acquisition parameters throughout the data collection.

In the past, CMR simulations have been used within a limited scope. Simulations have been used to identify the effect of various acquisition parameters on measurement accuracy and precision $[10,16]$ and to produce T1-maps through an inverse problem solving fitting procedure [17]. However, in order for the simulator to be executed within a reasonable amount of time, several assumptions and compromises on pulse sequence design had to be made (such as the addition of a crusher to reduce T2dependency). For the same reason some realistic aspects of the experiment (such as excitation slice profile) had not been incorporated.

In this study, we propose Simulations for QUAntifying RElaxation Magnetic Resonance constants (SQUAREMR), a new method for extracting quantitative tissue MR data from clinical pulse sequences with the aid of comprehensive, parallel MRI simulations of the Bloch equations. The specific aim was to utilize realistic simulations of MOLLI on a large population of spins so as to compute all possible outcomes of this pulse sequence for a range of physiologically relevant tissue relaxation constants. We hypothesized that quantitative CMR acquired with MOLLI can be improved by comparing the signals acquired from the MRI scanner to the entire pool of possible outcomes that are produced by these simulations for different tissue types. While this study is focused on a MOLLI-based T1 mapping technique, it could however be extended in other types of quantitative MRI throughout the body.

\section{Methods}

\section{MOLLI theory and pitfalls}

Figure 1 demonstrates a basic MOLLI pulse sequence scheme [5], where two inversion-recovery-prepared modified-Look-Locker experiments are separated by a pause, which is usually defined in terms of a number of heart cycles. In short, each modified-Look-Locker experiment consists of ECG-triggered single-shot acquisitions performed at end-diastole of consecutive heart beats. Each single-shot acquisition consists of a ramp up preparation (a startup sequence where the flip angle is increased linearly) followed by a balanced steady-state free precession (bSSFP) readout. For every modifiedLook-Locker experiment, the effective inversion times (TIs) of the single-shot images are defined by the time measured between the end of the inversion recovery (IR) radiofrequency pulse and the center of k-space of each bSSFP readout within the same modified-Look-Locker experiment. At the end of the MOLLI experiment, the acquired images undergo exponential fitting on a pixelby-pixel basis in order to estimate T1.

In MOLLI T1 mapping, the bSSFP readouts that follow the IR pulse perturb the relaxation process itself and do not allow for an ideal exponential recovery that is based solely on the equilibrium magnetization (M0) and the relaxation constant $\mathrm{T} 1$. As a result, the true recovery follows an apparent relaxation constant $\mathrm{T} 1 *$ which is always shorter than the true relaxation constant $\mathrm{T} 1$. MOLLI reconstruction compensates for this apparent relaxation by means of a 3-parameter exponential signal model and the "Look-Locker" correction factor [5]. However, the "Look-Locker" correction factor has been derived based on a Fast Low Angle Shot (FLASH) readout [18] and is used for the bSSFP readout since a simple closed form expression does not exist for the actual MOLLI pulse sequence. Although it has been shown that the FLASH-based correction factor is reasonably effective under specific conditions [10], it does lead to inaccuracies in T1 maps. 




Fig. 1 MOLLI acquisition scheme 5(3p)3. Illustration of a MOLLI pulse sequence scheme consisting of two successive ECG-triggered modifiedLook-Locker experiments (modified LL1 and modified LL2) with five and three single-shot readouts respectively. A pause of three heart beats has been retained between the two modified-Look-Locker experiments. Each single-shot acquisition consists of a ramp up preparation followed by a balanced steady-state free precession (bSSFP) readout

\section{SQUAREMR overview}

The basic concept of this study is based on the premise that the signals obtained from the simulation of a clinical pulse sequence on simulated tissue with specific relaxation constants ( $\mathrm{T} 1, \mathrm{~T} 2)$ would be identical to the measured signals acquired from the MRI scanner by applying the same pulse sequence on real tissue with the same relaxation constants (T1, T2). Therefore, for the same pulse sequence, obtaining identical simulated and measured signals would ideally indicate that the tissue constants were identical in both the simulation and the MRI scanner. Using a closed form expression of the clinical pulse sequence was not necessary but instead the identical pulse sequence was simulated and was applied on a computer model of spins. The solutions of the Bloch equations provided the temporal evolution of each spin magnetization vector under the influence of the RF pulses and magnetic field gradients of the pulse sequence.

A basic block diagram of SQUAREMR is shown in Fig. 2. In a conventional manner (dashed lines), the patient is scanned in an MRI scanner by applying a userselected acquisition parameter set. Then, the MRI signals, acquired from the MRI scanner, are processed in order to quantify tissue relaxation constants. With SQUAREMR, several steps were added to this workflow (solid lines). The identical acquisition parameter set was used by a parallel simulator to process the entire range of physiological tissue relaxation constants. The noisefree simulated signal was sampled at the exact inversion times (TIs) used in the MRI scanner with MOLLI. Then, a custom database of simulated signals was constructed so as to link each recorded simulated signal to a single pair of T1 and T2 values. Then, for the MOLLI images acquired by the scanner, on a pixel-by-pixel basis, the relaxation times $\mathrm{T} 1$ and $\mathrm{T} 2$ were estimated by finding the simulated signal in the database that produced the least squared difference to the MOLLI MRI signal within a pixel. In other words, when this least squared difference was identified, the related database entry returned the tissue relaxation constants.

\section{Acquisition parameters}

The acquisition parameters described the MOLLI pulse sequence. These included the following parameters: number of inversion-recovery-prepared modified-LookLocker experiments, number of ECG-triggered singleshot acquisitions per modified-Look-Locker experiment, number of pausing heart cycles between modified-LookLocker experiments, IR RF pulse duration and shape, TR and TE durations, bSSFP readout RF pulse duration and shape, slice thickness, acquisition scheme, Field-Of-View

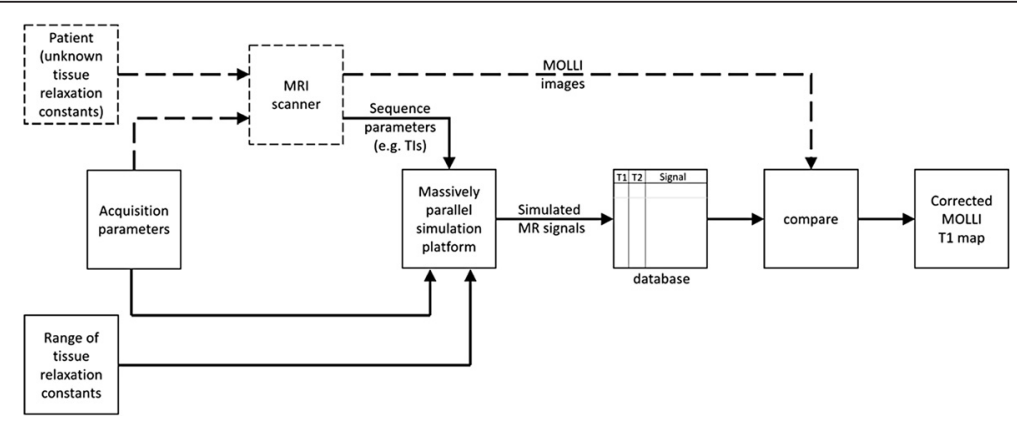

Fig. 2 Block diagram of SQUAREMR. The dashed lines demonstrate the conventional MOLLI workflow whereas solid lines illustrate the workflow steps introduced by SQUAREMR. (HR= heart rate) 
(FOV), matrix size, SENSE factor, receiver bandwidth (rBW) and number of startup TRs in the linear flip angle ramp.

In MOLLI, the TIs for a number of the single-shot images depend on the actual heart rate and cannot be known before the pulse sequence execution. Therefore, the simulations were performed after image acquisition.

\section{Parallel simulation platform}

A comprehensive MR physics simulator, MRISIMUL, was used $[19,20]$. Compared to other previously developed MR simulators, MRISIMUL is neither an imagebased simulator that utilizes T1, T2 and PD maps in combination with equations that describe the image intensity [21] nor a kspace-based simulator that utilizes the k-space formalism [22]. MRISIMUL is a simulation platform based on discrete-event Bloch equations applied on anatomical models of spins that incorporates realistic aspects of the MR experiment, makes no assumptions or simplifications for simulating the underlying MR physics and exploits parallel computing based on Graphics Processing Units (GPUs) for high computational performance. The computationally demanding core services (kernel) of MRISIMUL were developed in CUDA-C (NVIDIA, Santa Clara, CA) and distributed in parallel within the graphic processing units (GPUs) whereas the simulation wrapper was developed in MATLAB (The Mathworks Inc., Natick, MA, USA).

For each MOLLI experiment, the identical pulse sequence was simulated on a population of spins for a large range of physiological combinations of T1 and T2. T1 and T2 values of $200-1900 \mathrm{msec}$ and $20-400 \mathrm{msec}$ respectively were simulated with a step of $1 \mathrm{msec}$. Simulations were not performed for combinations where T2 > T1. To explore faster SQUAREMR execution times, a step of $5 \mathrm{msec}$ was also used. The ranges of T1 and T2 values were chosen based on physiological myocardial and blood values found in the literature $[10,14]$ with an expanded range based on the characteristics of each experiment. For example, since no gadolinium was administered, very short $\mathrm{T} 1$ values below $200 \mathrm{msec}$ were not considered. For each one of the simulations, the MOLLI pulse sequence was applied on a spin with unique characteristics (T1, T2 and position along the slice direction). The simulated MOLLI pulse sequence was based on the pulse sequence run on the MRI scanner. The receiver bandwidth (rBW) of the MRI scanner also defined the temporal step $\Delta t$ of the simulated pulse sequence. A total of approximately 75,000 to 150,000 time steps were computed for each simulation. The Bloch simulation temporal resolution was $10 \mu \mathrm{sec}$ and $5 \mu \mathrm{sec}$ respectively. The bSSFP condition was retained throughout the simulated MOLLI pulse sequence, whereas software crushers [19] were utilized before and after the IR pulse. Also, in order for realistic slice profiles to be incorporated in the simulations, approximately 20 to 100 spins were simulated across the slice thickness. A total of approximately 532,000 to $63,400,000$ simulations of the entire imaging pulse sequence were performed. The resulting database consisted of a total of approximately 25,000 to 628,000 entries respectively.

Simulations were performed on a single-node system consisting of a server style computer of 2 hexa-core (Intel E5-2630, $2.30 \mathrm{GHz}$ ) processors, $32 \mathrm{~GB}$ RAM and four Tesla C2075 GPU cards. Each Tesla C2075 graphics card utilized a total dedicated memory of 6 GB GDDR5 and a total of 448 stream processors.

\section{SQUAREMR performance}

To investigate SQUAREMR performance, two sets of pre-Gd MOLLI experiments were performed. For a given experiment, the total SQUAREMR execution time could be reduced by decreasing either the total number of spins within the spin model or the total number of pulse sequence time steps or the T1 map size or a combination of them.

The first set of MOLLI experiments was performed with a simulation temporal step of $10 \mu \mathrm{sec}$ and an acquisition matrix size of $128 \times 128$ whereas the second set of experiments was performed with a simulation temporal step of $5 \mu \mathrm{sec}$ and an acquisition matrix size of $320 \times 320$. To further investigate how spin model size relates to SQUAREMR performance, two different test cases were considered for each set of experiments. For the first test case, a short T1 and T2 sampling step of $1 \mathrm{msec}$ was combined with a large number of 101 spins along slice thickness resulting in a total of approximately 533,000 database entries. For the second test case, a longer T1 and T2 sampling step of $5 \mathrm{msec}$ was combined with a small number of 21 spins along slice thickness resulting in a total of approximately 21,500 database entries. For both test cases, T1 and T2 values of 600-2000 msec and 20-400 msec respectively were simulated.

\section{Phantom setup}

Two phantoms of six "tissues", each with its own T1 and $\mathrm{T} 2$ values, were used in this study. The phantoms were prepared with varying concentrations of $\mathrm{CuSO}_{4}$ and Agar [23] in order to obtain specific combinations of T1 and T2 values. Agarose powder was weighted and dissolved in distilled water and the proper amount of a $10 \mathrm{mM} \mathrm{CuSO}_{4}$ solution was added. The mixture was heated, poured into containers (one per "tissue") and was left to reach room temperature.

The first phantom consisted of six "tissues" (plastic bottles of $500 \mathrm{ml}$ ) with target $\mathrm{T} 1$ and $\mathrm{T} 2$ values close to real cardiac tissues relaxation constants found in the literature, both with and without gadolinium contrast 


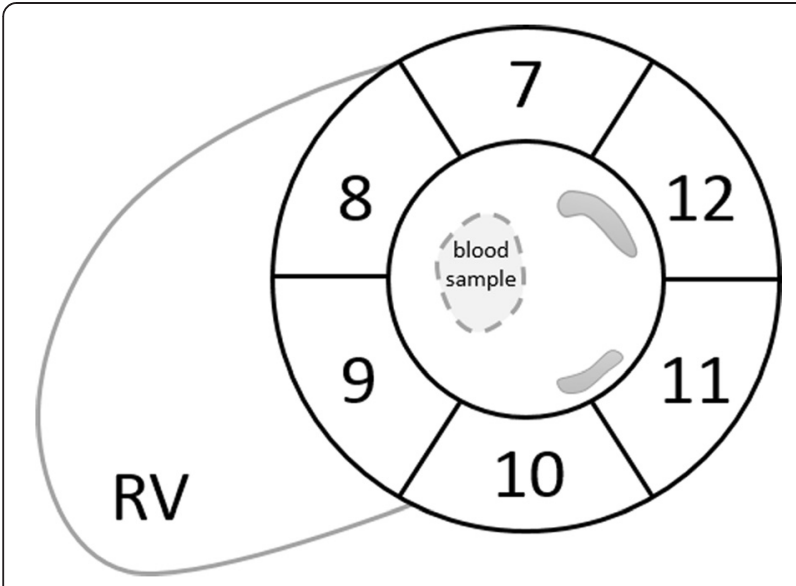

Fig. 3 Myocardial segmentation and blood region of interest in a mid-ventricular short-axis slice. Display of the six myocardial segments [32] and the region of interest within the blood pool from which blood relaxation constants were measured (dashed area)

agent present. The "tissues" used in this set had T1s and T2s of pre-contrast normal myocardium [11, 24], precontrast blood [10, 25], pre-contrast edematous myocardium [26], pre-contrast infarcted myocardium [5, 24], post-contrast normal myocardium (2-3 min after gadolinium contrast administration) and post-contrast normal myocardium (13-15 min after contrast administration) $[27,28]$. The second phantom set consisted of 6 "tissues" (Eurospin II Test System, Livingston, UK) with $\mathrm{T} 2$ values close to $\mathrm{T} 2$ of normal myocardium [24] and $\mathrm{T} 1$ values covering the range from $200 \mathrm{msec}$ to 1600 msec.

To study the SQUAREMR T2 estimates obtained with the MOLLI sequence a third set of 10 phantoms of varying combinations of $\mathrm{T} 1$ and $\mathrm{T} 2$ values was scanned with the clinically relevant $5(3 p) 3$ scheme only. The target T1 and T2 values were chosen so as to cover the following four combinations: short T1 and short T2 values; short $\mathrm{T} 1$ and long $\mathrm{T} 2$ values; long $\mathrm{T} 1$ and short $\mathrm{T} 2$ values; long $\mathrm{T} 1$ and long $\mathrm{T} 2$ values.

Relaxation constants reference standard values were measured on a $1.5 \mathrm{~T}$ Philips Achieva systems (Philips
Healthcare, Best, Netherlands). For T1 measurements Saturation Recovery was used (Tsat $=0.01-15 \mathrm{sec}$, $\mathrm{TR}=10 \mathrm{sec}$ ) because it allowed for visual evaluation of the effectiveness of the saturation pulse at the shortest saturation time i.e. for long T1s, the remaining signal was within or uniformly close to the noise floor. For T2 measurements T2p-SSFP was used for faster data acquisition since it has been validated in the past as a reference standard against slow spin-echo experiments [29].

\section{Healthy volunteer population}

Twelve (12) healthy volunteers with no medical history (12 men, age $34 \pm 12$ years) were studied. Eight out of twelve were studied with the clinically relevant $5(3 p) 3$ scheme only. The study was approved by the local ethics committee and all subjects provided written consent (The regional ethics committee, Lund, Sweden. Ethics application number: 541/2004).

\section{CMR protocol}

CMR studies were performed on a 1.5 T Philips Achieva scanner (Philips Healthcare, Best, Netherlands) equipped with a 32-channel receiver coil and advanced research packages for cardiac applications. The CMR protocol included a series of MOLLI pulse sequences with different acquisition schemes:

1. 5(3p)3 (clinical MOLLI pulse sequence for pre-contrast myocardial T1 mapping) $[9,10]$

2. 4(1p)3(1p)2 (clinical MOLLI pulse sequence for post-contrast myocardial T1 mapping) [10]

3. 5(0p)3 (custom MOLLI pulse sequence for shorter myocardial T1 mapping acquisition)

where $\mathrm{p}$ stands for heartbeats pauses between modifiedLook-Locker experiments (Fig. 1).

All MOLLI pulse sequences shared the following parameters: the IR pulse was a hyperbolic secant adiabatic pulse [30] with $4.74 \mathrm{msec}$ duration, whereas the bSSFP readout used a $490 \mu \mathrm{sec}$ sinc shaped RF pulse with $6 \mathrm{~mm}$ slice thickness and $35^{\circ}$ excitation flip angle, rBW $200 \mathrm{kHz}$ (1612.9 Hz/pixel), field-of-view (FOV) $272 \mathrm{~mm} \times 272 \mathrm{~mm}$,

Table 1 Reference T1 and T2 values of the "tissues" in the myocardium phantom

\begin{tabular}{|c|c|c|c|c|c|c|}
\hline Tissue type & $\mathrm{T} 1$ [ms] & T1 StDev [ms] & $\mathrm{T} 2$ [ms] & T2 StDev [ms] & CuSO4 [g/L] & Agarose $[\mathrm{g} / \mathrm{L}]$ \\
\hline Normal myocardium (pre-contrast) & 1048 & 12 & 50 & 2 & 0,12 & 17.80 \\
\hline Blood (pre-contrast) & 1570 & 20 & 196 & 11 & 0,07 & 4.00 \\
\hline Edema (pre-contrast) & 1249 & 15 & 62 & 3 & 0,09 & 14.67 \\
\hline Infarct (pre-contrast) & 1361 & 21 & 64 & 3 & 0,07 & 14.51 \\
\hline Normal myocardium (post-contrast 2-3 min) & 344 & 6 & 52 & 4 & 0,2 & 20.00 \\
\hline Normal myocardium (post-contrast 13-15 min) & 413 & 12 & 50 & 3 & 0,5 & 17.51 \\
\hline
\end{tabular}

T1 and T2 values were measured with Saturation Recovery and T2p-SSFP respectively on a 1.5 T Philips Achieva systems (Philips Healthcare, Best, Netherlands) 


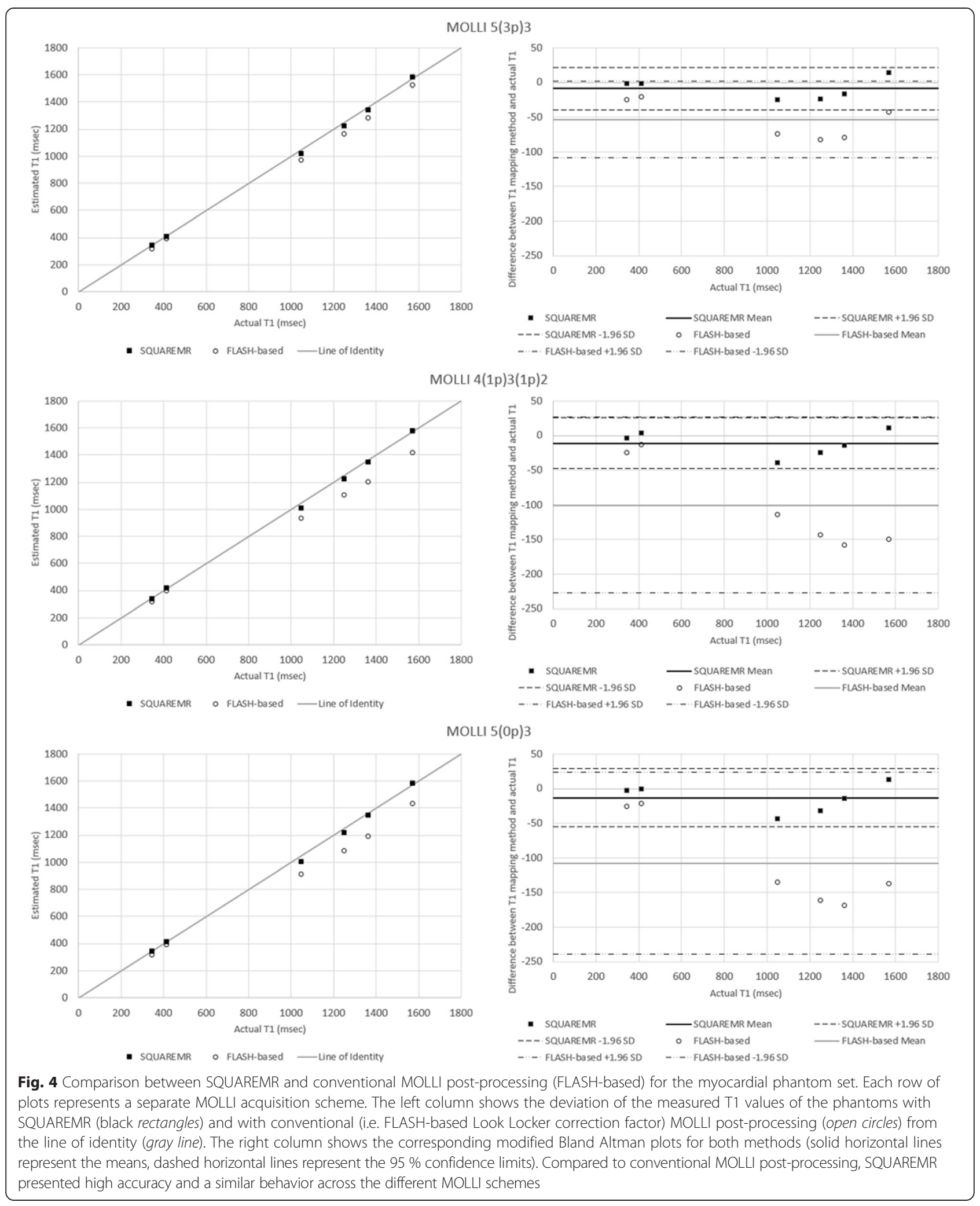




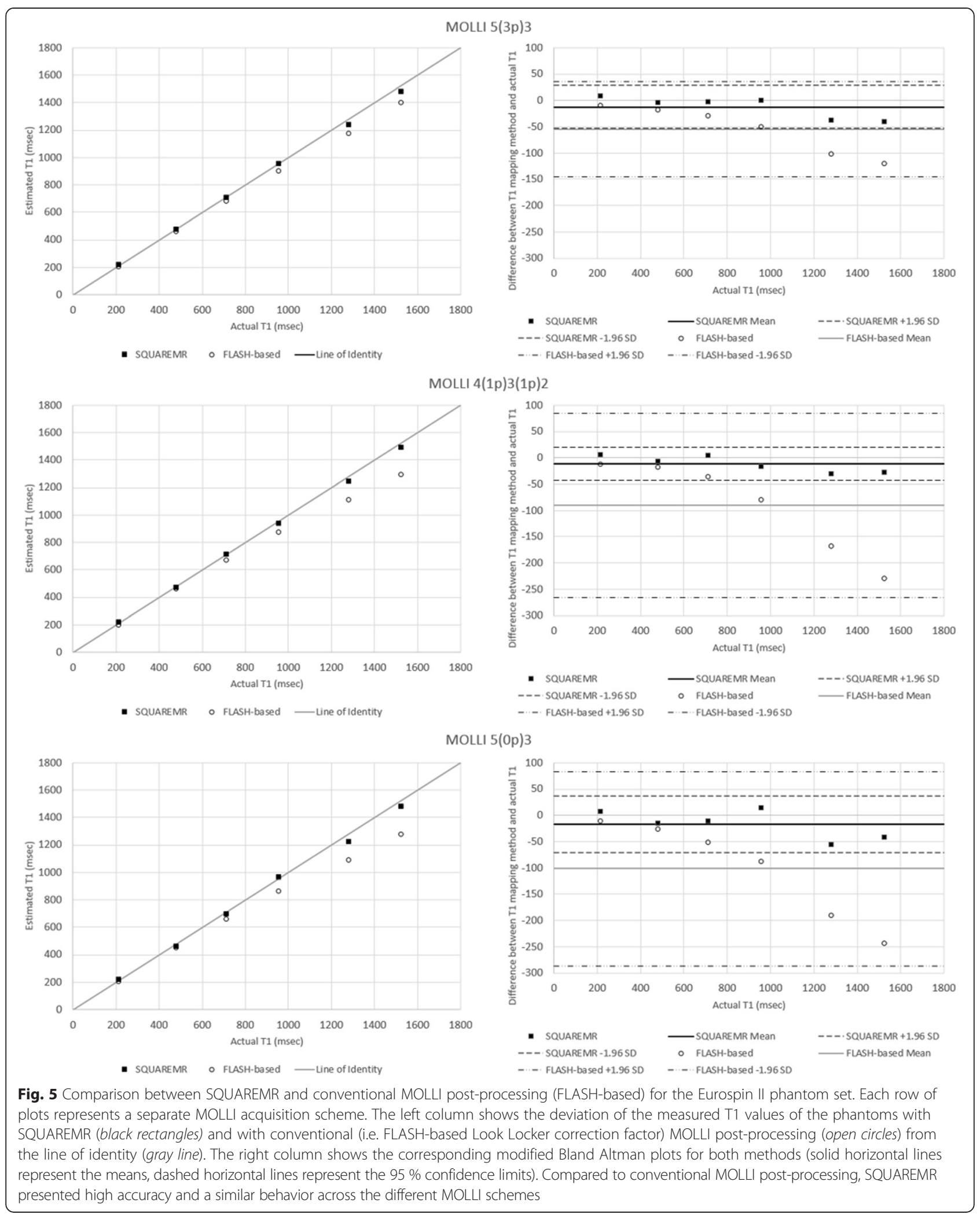


acquisition matrix $124 \times 124$, linear k-space trajectory and SENSE acceleration factor of 2 (actual number of phase encoding steps was 65). A linear ramp up preparation of 10 pulses was used to reach steady state prior to the bSSFP readout.

In phantom studies TR was set to $2.54 \mathrm{msec}$ and TE to $1.27 \mathrm{msec}$. MOLLI schemes 1 and three used initial TIs equal to $114 \mathrm{msec}$ and $350 \mathrm{msec}$ whereas MOLLI scheme 2 used initial TIs equal to $114 \mathrm{msec}, 232 \mathrm{msec}$ and $350 \mathrm{msec}$ (initial TI increment of $118 \mathrm{msec}$ ). For phantoms, a 6-channel head coil was used along with a simulated ECG (60 beats per minute). A coronal single slice was acquired for the myocardial phantom whereas an axial single slice was acquired for the Eurospin II phantom.

In healthy volunteer studies the TR was $3 \mathrm{msec}$ and the TE $1.5 \mathrm{msec}$. MOLLI schemes 1 and 3 used initial TIs of $134 \mathrm{msec}$ and $350 \mathrm{msec}$ whereas MOLLI scheme 2 used initial TIs of $134 \mathrm{msec}, 242 \mathrm{msec}$ and $350 \mathrm{msec}$ (initial TI increment of $108 \mathrm{msec}$ ). The CMR protocol was applied in a single mid-ventricular short axis slice with a 32-channel receiver coil.

The term "initial TI" was defined as the first TI measured between the end of the adiabatic inversion pulse and the center of $\mathrm{k}$-space of the first single-shot bSSFP readout that followed within the same modified-LookLocker experiment. The TIs between the inversion pulse and the center of the other bSSFP readouts within the same modified-Look-Locker experiment were determined by the initial TI and the duration of the cardiac cycles preceding each readout.

\section{Image analysis}

Parameter mapping with SQUAREMR was performed using the GPU-framework of MATLAB (The Mathworks Inc., Natick, MA, USA) on a single GPU whereas MOLLI $\mathrm{T} 1$ values were measured from the MOLLI magnitude images using conventional MOLLI post-processing (i.e. with a FLASH-based Look Locker correction factor $[5,18])$ with a 3-parameter fit $[5,10]$. In-vivo myocardial segmentation was implemented manually [31]. For phantom studies, relaxation constants were measured by placing a rectangular region of interest (ROI) in the center of each phantom and estimated T1 values were reported. For the third set of phantoms, which was intended for studying the T2 estimates, both T1 and T2 values were reported. In healthy volunteer studies, left-ventricular (LV) myocardium was segmented in 6 areas in the mid-ventricular



Fig. 6 SQUAREMR T1 and T2 estimates of ten phantoms in comparison to their reference T1 and T2 values for a clinically relevant 5(3p)3 MOLLI acquisition scheme. In black rectangles are the T1 and T2 values given by SQUAREMR whereas in black $\mathrm{x}$ are the corresponding relaxation constants reference standard values. SQUAREMR demonstrated a small T1 estimation error for all T1 and T2 combinations. The T2 estimation error was small only for phantoms with long $\mathrm{T} 1$ and short $\mathrm{T} 2$ values. For samples with other $\mathrm{T} 1$ and $\mathrm{T} 2$ combinations, SQUAREMR demonstrated a larger error in estimating T2 with the MOLLI pulse sequence 


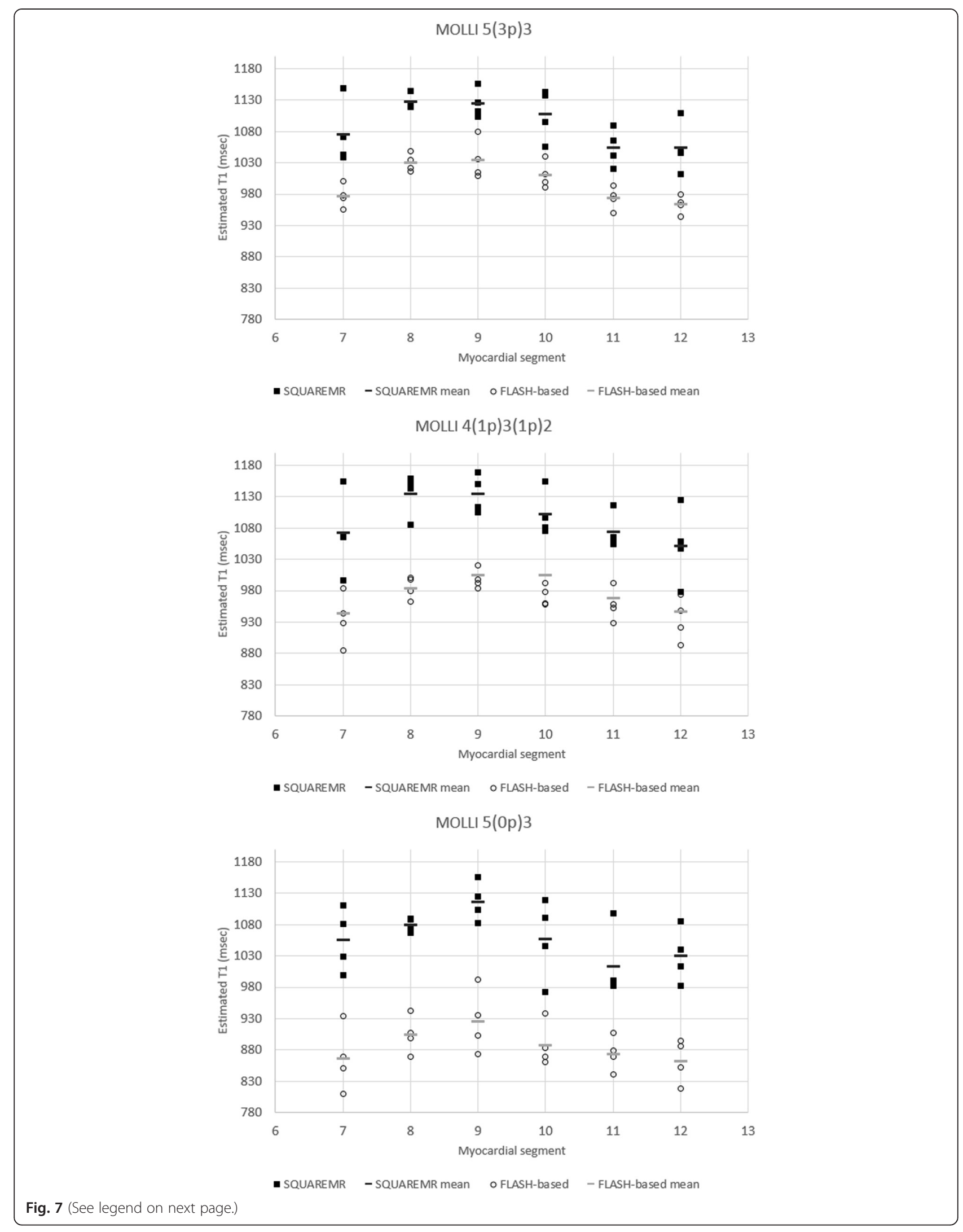


(See figure on previous page.)

Fig. 7 Myocardial T1 values measured from the six myocardial segments (7 to 12). Myocardial T1 values were measured in the mid-ventricular short-axis slices for three different MOLLI acquisition schemes. In black rectangles are the T1 values given by SQUAREMR whereas in black circles are the corresponding T1 values given by the conventional MOLLI post-processing (FLASH-based). In solid black and gray short lines are the mean segmental T1 values for SQUAREMR and conventional MOLLI post-processing respectively. Data were extracted from the four volunteers that were examined additionally with 4(1p)3(1p)2 and 5(0p)3

short axis (SAX) slice [32] and relaxation times were measured for each segment separately but also for the entire LV myocardium. Blood T1 and T2 values were measured from an ROI placed within the LV blood pool (Fig. 3). Myocardium and blood ROIs were drawn so as to avoid signal contamination from adjacent tissues. All values in this study are reported as mean \pm standard deviation (SD). For phantom studies, modified Bland-Altman plots [33] were used to demonstrate the agreement of the two methods (SQUAREMR and conventional MOLLI postprocessing) with the reference standards.

\section{Statistics}

Comparisons were performed with student's two tailed $t$-test for paired data.

\section{Results}

\section{Phantom studies}

The reference $\mathrm{T} 1$ and T2 values of the "tissues" in the first phantom are shown in Table 1 . The reference T1 relaxation times of the "tissues" in the second phantom ranged from $212 \mathrm{msec}$ to $1522 \mathrm{msec}$ whereas the reference T2 relaxation times was kept close to that of normal myocardium (52 $\pm 6 \mathrm{msec})$.

Results from the first phantom are shown in Fig. 4. For the range of relaxation times corresponding to cardiac tissues (pre- and post- contrast) that were studied, SQUAREMR presented high accuracy and a similar behavior across the different MOLLI schemes. For the first phantom, SQUAREMR demonstrated better accuracy compared to conventional MOLLI post-processing [5(3p)3 scheme bias of $8.8 \pm 15.3 \mathrm{msec}$ vs. $53.6 \pm 28.2 \mathrm{msec}$, $p<0.05 ; 4(1 \mathrm{p}) 3(1 \mathrm{p}) 2$ scheme bias of $11 \pm 18.8 \mathrm{msec}$ vs. $100.2 \pm 64.9$ msec, $p<0.05 ; 5(0 \mathrm{p}) 3$ scheme bias of $13.1 \pm 21.1 \mathrm{msec}$ vs. $107.9 \pm 66.9 \mathrm{msec}, p<0.05 ; N=6]$, even in cases of "tissues" with long T1s and MOLLI schemes that do not allow for full relaxation of long $\mathrm{T} 1 \mathrm{~s}$ prior to the next inversion. Conventional MOLLI postprocessing in these cases presented a bias higher than $100 \mathrm{msec}$ for the phantoms with high T1 values.

In the second phantom, which had close to normal myocardium T2s and a range of T1s, SQUAREMR demonstrated better accuracy than conventional MOLLI [5(3p)3 scheme bias of $12.1 \pm 20.5 \mathrm{msec}$ vs. $54.7 \pm$ 45.7 msec, $p<0.05$; $4(1 \mathrm{p}) 3(1 \mathrm{p}) 2$ scheme bias of $11.4 \pm$ $16.1 \mathrm{msec}$ vs. $90.2 \pm 89.3 \mathrm{msec}, p<0.05$; 5(0p)3 scheme bias of $16.9 \pm 27.2 \mathrm{msec}$ vs. $101.3 \pm 94.3 \mathrm{msec}, p<0.05$;
$N=6$ ], (Fig. 5). Conventional MOLLI post-processing introduced an increasing error with increasing T1, as has been previously shown in simulation studies [10].

Figure 6 shows the SQUAREMR T1 and T2 estimates in phantoms $(N=10)$ against their reference $\mathrm{T} 1$ and reference T2 values. SQUAREMR demonstrated small error $(14.3 \pm 11.2 \mathrm{msec} ; N=10)$ in estimating $\mathrm{T} 1$ for all $\mathrm{T} 1$ and T2 combinations. The error was also relatively small in estimating T2 $(7.2 \pm 6 \mathrm{msec} ; N=4)$ for phantoms with high $\mathrm{T} 1$ and low T2 values. For other T1 and T2 combinations, SQUAREMR yielded larger errors $(45.3 \pm 31.8 \mathrm{msec}$; $N=6$ ) in estimating $\mathrm{T} 2$.

\section{Human studies}

Figure 7 shows the segmental T1 values given by SQUAREMR and the conventional MOLLI postprocessing for the three different MOLLI schemes. SQUAREMR yielded higher T1 values for all segments compared to the conventional MOLLI post-processing, for all three MOLLI schemes. With the MOLLI scheme $5(3 p) 3$, which is being used clinically for pre-contrast myocardial T1 mapping, the average T1 value per short axis slice was $1025 \pm 22.9 \mathrm{msec}$ for conventional MOLLI post-processing and $1117 \pm 25.6 \mathrm{msec}$ for SQUAREMR $(p<0.001, N=12)$. The average myocardial $\mathrm{T} 1$ value for segment 9 (inferior septal myocardium) was $1053 \pm$ $33 \mathrm{msec}$ for conventional MOLLI post-processing and $1148 \pm 38 \mathrm{msec}$ for SQUAREMR $(p<0.001, N=12)$. The average blood T1 values were $1570 \pm 52.8 \mathrm{msec}$ and $1634 \pm 50 \mathrm{msec}$ respectively $(p<0.001, N=12)$. As other studies have already shown $[6,14]$, a regional variation of segmental average T1 values was measured whereas the average $\mathrm{T} 1$ value of myocardial segment 9 was higher compared to the average T1 value of the entire slice.

Figure 8 shows how the T1 values, resulting from SQUAREMR and from the conventional MOLLI post processing, have been affected by the three different MOLLI schemes. The T1 values were measured over the entire slice, myocardial segment 9 and blood pool. SQUAREMR was well behaved with consistent mean T1 values and consistent variance across the different MOLLI schemes. The conventional MOLLI post-processing overall showed significantly different mean $\mathrm{T} 1$ values between schemes [e.g. $p<0.001$ for myocardial $5(3 p) 3$ vs. 5(0p)3]. Table 2 gives the results presented in Fig. 8 whereas Fig. 9 shows T1 maps from a healthy volunteer derived from the conventional MOLLI post processing (left image) and 


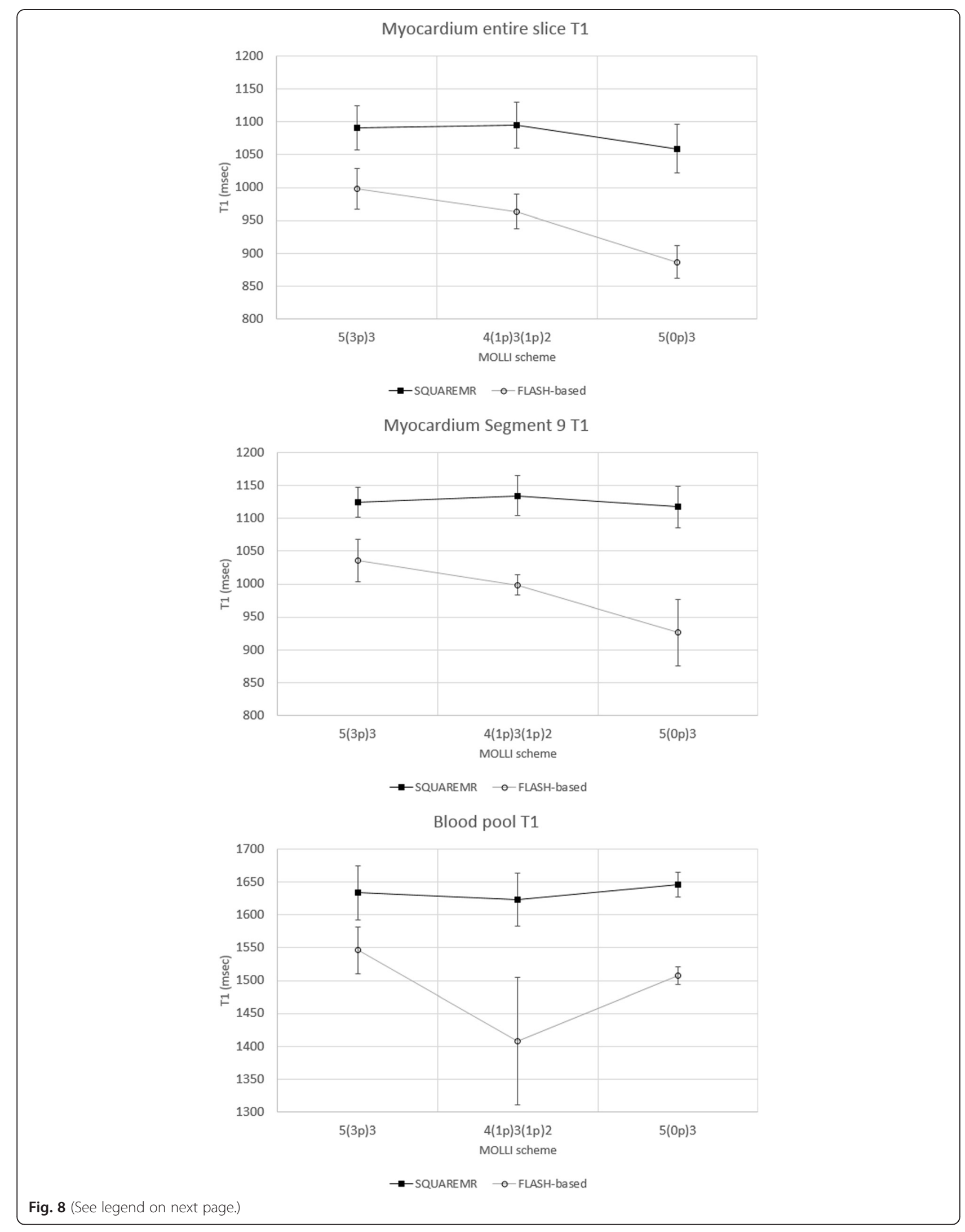


(See figure on previous page.)

Fig. 8 Mean T1 values per short axis slice, myocardial segment nine and blood pool. The mean T1 values were measured with SQUAREMR and conventional MOLLI post-processing for three different MOLLI acquisition schemes. In black rectangles are the mean T1 values given by SQUAREMR whereas in black circles are the corresponding mean T1 values given by the conventional MOLLI post-processing (FLASH-based). Error bars depict the standard deviation of the mean for each MOLLI acquisition scheme. Data were extracted from the four volunteers that were examined additionally with $4(1 \mathrm{p}) 3(1 \mathrm{p}) 2$ and 5(0p)3

SQUAREMR (right image) for a 5(3p)3 acquisition scheme.

\section{SQUAREMR performance}

To illustrate the performance of SQUAREMR, two different sets of pre-Gd MOLLI experiments were examined. The simulation execution times and the database search times for different MOLLI experiment sizes were recorded and are shown in Table 3. It can be seen that the duration of the SQUAREMR processing varied depending on the complexity of the experiment, which can be defined by a number of parameters such as the size of the acquisition matrix, the number of database entries, MOLLI pulse sequence timesteps, etc. The total execution times range from $33 \mathrm{~s}$ to $47 \mathrm{~min}$ on a server with four Tesla C2075 GPU cards.

\section{Discussion}

A new method for improving measurements from clinical pulse sequences in CMR relaxometry was presented. The use of parallel simulations was shown to improve the T1 estimates in MOLLI by comparing the MRI signals acquired from the MRI scanner to the entire pool of physiological simulated signals that were produced by parallel simulations of the identical pulse sequence on a population of spins. While the current study explored the feasibility of obtaining T1 properties from MOLLI images, in principle it could be extended in other areas of quantitative MR.

MOLLI T1 mapping is widely used today; however the correction of the T1 recovery relies on the a FLASH closed form expression [5] only because such an expression does not exist for the actual bSSFP readout that the MOLLI pulse sequence utilizes. Several studies have already shown that MOLLI T1 mapping underestimates true $\mathrm{T} 1$ whereas its measurement error is influenced by several acquisition protocol parameters $[5,6,10,12,16]$. SQUAREMR does not rely on closed form expressions but rather on an extended simulation of the pulse sequence itself. The basic concept of SQUAREMR was based on the premise that realistic simulations of clinical pulse sequences on tissue models of specific parameters (T1, T2) would result in identical signals to the signals acquired from the MRI scanner after the application of the same pulse sequences on true tissues with the same relaxation properties.

In the current work, a CMR protocol consisting of three different MOLLI pulse sequences was implemented: 5(3p)3, 4(1p)3(1p)2 and 5(0p)3. For every MOLLI experiment, the identical MOLLI pulse sequence was simulated for T1 and T2 ranges of 200 to $1900 \mathrm{msec}$ and of 20 to $400 \mathrm{msec}$ respectively taking into account realistic aspects of the MR experiment, such as realistic excitation slice profiles and heart rate variation in in-vivo studies. For that purpose, MRISIMUL, a GPUbased, MR physics simulator $[19,20]$ was utilized in this study.

The CMR T1 mapping protocol was applied on three phantom setups and on twelve healthy volunteers. All twelve volunteers and phantom setups were examined with the clinical 5(3p)3 scheme. The actual TI timings were extracted from the scanner data and used for the SQUAREMR simulations allowing for more realistic simulations. Four out of twelve volunteers and two out of three phantom setups were imaged additionally with $4(1 p) 3(1 p) 2$ and 5(0p)3. Results of the CMR protocol on the phantom setups demonstrated improvement of accuracy and a substantially reduced T1 variability across the different MOLLI schemes. Compared to conventional MOLLI post-processing, SQUAREMR showed improved accuracy even for long T1s with no pause between modified-Look-Locker experiments. The latter

Table 2 Mean T1 values (msec) per slice, myocardial segment 9 and blood pool

\begin{tabular}{|c|c|c|c|c|c|c|c|c|c|c|c|c|}
\hline \multirow[b]{3}{*}{ MOLLI scheme } & \multicolumn{4}{|c|}{ Myocardium entire slice T1 } & \multicolumn{4}{|c|}{ Myocardium segment $9 \mathrm{~T} 1$} & \multicolumn{4}{|c|}{ Blood pool T1 } \\
\hline & \multicolumn{2}{|l|}{$\overline{\mathrm{MOLLI}}$} & \multicolumn{2}{|c|}{ SQUAREMR } & \multicolumn{2}{|l|}{$\overline{\mathrm{MOLLI}}$} & \multicolumn{2}{|c|}{ SQUAREMR } & \multicolumn{2}{|l|}{$\overline{\text { MOLLI }}$} & \multicolumn{2}{|c|}{ SQUAREMR } \\
\hline & Mean & SD & Mean & SD & Mean & SD & Mean & SD & Mean & SD & Mean & SD \\
\hline $5(3 p) 3$ & 998 & 31 & 1091 & 34 & 1035 & 32 & 1125 & 23 & 1546 & 36 & 1633 & 41 \\
\hline $4(1 p) 3(1 p) 2$ & 964 & 26 & 1095 & 35 & 998 & 16 & 1135 & 30 & 1408 & 97 & 1623 & 40 \\
\hline $5(0 p) 3$ & 887 & 25 & 1059 & 37 & 926 & 51 & 1117 & 31 & 1507 & 13 & 1646 & 19 \\
\hline
\end{tabular}

T1 values were measured with SQUAREMR and conventional MOLLI post-processing for three different MOLLI acquisition schemes. Data were extracted from the four volunteers that were examined additionally with $4(1 p) 3(1 p) 2$ and $5(0 p) 3$ 




Fig. 9 T1 maps of a healthy volunteer. Left image: Conventional MOLLI map derived from a clinical pulse sequence. Right image: T1 map obtained from MOLLI-based tissue signal intensities using SQUAREMR. (MOLLI acquisition scheme 5(3p)3, rBW=200 kHz, FOV= $272 \mathrm{~mm} \times 272 \mathrm{~mm}$, acquisition matrix $=124 \times 124$, SENSE acceleration factor $=2$ ). Grayscale images are presented to avoid exaggerating contrast when crossing different color boundaries, which commonly occurs with color images

demonstrates that the T1 estimates are not dependent on the duration of the delay between successive modified Look-Locker experiments, which in patient scans may change due to heartrate variations.

In order to investigate whether SQUAREMR could also provide T2 estimates from data obtained with a MOLLI pulse sequence, the methodology was applied on phantoms with a range of $\mathrm{T} 1$ and $\mathrm{T} 2$ values. SQUAREMR showed reasonable T2 values in phantoms with long T1s and short T2s. Larger errors in estimating T2 were observed for the remaining T1 and T2 combinations; however, this was expected for a T1 mapping pulse sequence, such as MOLLI. Previous studies have shown that the MOLLI pulse sequence introduces some T2 modulation on the signal, which is more pronounced for long $\mathrm{T} 1$ and short T2 values [10, 34]; therefore, for these values SQUAREMR provided reasonable T2 estimates. For other T1s and T2s, SQUAREMR was unable to provide reasonable T2 estimates since the MOLLI signal simply did not contain this information. The T2 results shown in Fig. 6 indicate that this was not a limitation of SQUAREMR in terms of convergence but rather a limitation imposed by the MOLLI pulse sequence itself which has been designed to mainly modulate the MOLLI signal based on the T1 values of the tissues irrespective of their T2 values.

In in-vivo studies, myocardial and blood $\mathrm{T} 1$ values were measured. The average $\mathrm{T} 1$ values obtained with the CMR protocol for both the conventional MOLLI post- processing and SQUAREMR are summarized in Table 2 for $N=4$. SQUAREMR showed substantially elevated T1 values for both myocardium and blood pool when compared to conventional MOLLI post-processing. Conventional MOLLI T1 values in this work were similar to values previously reported in the literature. In particular, the conventional MOLLI scheme 5(3p)3 yielded an average blood T1 value of $1570 \pm 52 \mathrm{msec}(N=12)$. Previous studies have reported $1534 \mathrm{msec}$ with MOLLI [27] and $1516 \pm 21 \mathrm{msec}$ [13] with saturation recovery FLASH. Also, in this work, the conventional MOLLI T1 value for myocardium (segment 9) of $1053 \pm 33 \mathrm{msec}(N=12)$ was similar to that of previous studies with MOLLI $(1034 \pm 56 \mathrm{msec}$ [35] and $1052 \pm 41 \mathrm{msec}$ [11]). On the other hand, SQUAREMR resulted in MOLLI T1 values similar to more accurate CMR T1 mapping techniques. In particular, the average blood T1 value of $1634 \pm 50 \mathrm{msec}$ $(N=12)$ was close to that previously reported with SASHA $(1639 \pm 97 \mathrm{msec}[3])$. The average T1 value in myocardial segment nine obtained with SQUAREMR MOLLI was $1148 \pm 38 \mathrm{msec}(N=12)$ which was close to that previously reported with rapid cardiac gated singleshot IR-FSE sequence $(1092 \pm 64 \mathrm{msec}$ [8]) and two dimensional SASHA $(1105 \pm 46 \mathrm{msec}[36])$.

The application of a parallel realistic simulator in order to correct measured data from the scanner is a new concept. In the past, MR simulations have been used in a limited scope $[10,16,17]$ with the exception of

Table 3 SQUAREMR performance for MOLLI experiments with varying complexity

\begin{tabular}{|c|c|c|c|c|c|c|c|c|c|}
\hline$\#$ & $\begin{array}{l}\text { Simulation } \\
\text { timesteps }\end{array}$ & $\begin{array}{l}\text { Simulation temporal } \\
\text { step ( } \mu \text { sec) }\end{array}$ & $\begin{array}{l}\text { Database } \\
\text { entries }\end{array}$ & $\begin{array}{l}\text { Sampling step } \\
\text { (msec) }\end{array}$ & $\begin{array}{l}\text { T1 map } \\
\text { size }\end{array}$ & $\begin{array}{l}\text { Spins along } \\
\text { slice }\end{array}$ & $\begin{array}{l}\text { Simulation execution } \\
\text { (min:sec) }\end{array}$ & $\begin{array}{l}\text { Database search } \\
\text { (min:sec) }\end{array}$ & $\begin{array}{l}\text { Total execution } \\
\text { time (min:sec) }\end{array}$ \\
\hline 1 & 63705 & 10 & 533400 & 1 & $128 \times 128$ & 101 & $17 \mathrm{~m}: 47 \mathrm{~s}$ & $0 \mathrm{~m}: 29 \mathrm{~s}$ & $18 \mathrm{~m}: 16 \mathrm{~s}$ \\
\hline 2 & 63705 & 10 & 21560 & 5 & $128 \times 128$ & 21 & $0 \mathrm{~m}: 9 \mathrm{~s}$ & $0 \mathrm{~m}: 24 \mathrm{~s}$ & $0 \mathrm{~m}: 33 \mathrm{~s}$ \\
\hline 3 & 156877 & 5 & 533400 & 1 & $320 \times 320$ & 101 & $44 \mathrm{~m}: 27 \mathrm{~s}$ & $2 \mathrm{~m}: 47 \mathrm{~s}$ & $47 \mathrm{~m}: 14 \mathrm{~s}$ \\
\hline 4 & 156877 & 5 & 21560 & 5 & $320 \times 320$ & 21 & $0 \mathrm{~m}: 22 \mathrm{~s}$ & $1 \mathrm{~m}: 50 \mathrm{~s}$ & $2 \mathrm{~m}: 12 \mathrm{~s}$ \\
\hline
\end{tabular}

The simulation execution times and the database search times were recorded for the application of SQUAREMR on MOLLI experiment of varying size 
MR-fingerprinting [15]. SQUAREMR depends on extended and realistic MR simulations of already available clinical pulse sequences in order to build an extensive database of simulated signals identical (ideally) to the signals obtained from the MR scanner for the same experiment configuration. The utilization of multi-GPU technology along with the technology advancements taking place on the GPU hardware suggest that this method has the potential to become a real-time routine on the MRI scanner in the future.

In this work, some limitations apply. Simulation of magnetization transfer (MT) was not studied although previous simulation studies $[10,37]$ suggest that MT plays a role in T1 underestimation with MOLLI. However, accurate simulation of MT becomes challenging for the entire range of relaxation times being studied in this work since previous work has shown alteration of MT in disease cases (e.g. myocardial infarction [38]) and among different tissue types (blood vs. myocardium) [10]. Also, blood flow effects were not studied in this work. Simulations assumed a stationary spin model during the application of MOLLI pulse sequence. Although the bSSFP readout is applied during diastasis, when the heart muscle is mostly stationary, blood flow effects may alter the apparent $\mathrm{T} 1$ relaxation in the blood pool and, in turn, the T1 estimation [10]. In this implementation, a linear full search of the database was utilized, which limited the performance of SQUAREMR in terms of its execution speed. Non-linear optimization might be way forward but were not tested. In the future, optimization of algorithms for database construction (such as using a variable step for $\mathrm{T} 1$ and $\mathrm{T} 2$ values, eliminating database $\mathrm{T} 1, \mathrm{~T} 2$ pairs not pertaining to a particular MR application, etc.) and database search (such as using data specific schemes) could further improve overall SQUAREMR performance. Despite these limitations, SQUAREMR demonstrated improved T1 accuracy in phantom studies whereas in healthy volunteer studies the reported $\mathrm{T} 1$ values of myocardium and blood were close to those acquired with more accurate CMR T1 mapping techniques in the literature. Last but not least, the substantially reduced $\mathrm{T} 1$ variability across the different MOLLI schemes with SQUAREMR suggests that myocardial tissue characterization could potentially be achieved within 8 heart beats.

\section{Conclusion}

In conclusion, SQUAREMR is a new method that allows for quantification of CMR data with already available clinical pulse sequences and with the aid of comprehensive, parallel MRI simulations. In this study, a MOLLI-based T1 mapping example was investigated demonstrating improvement of accuracy in phantom studies and consistent mean $\mathrm{T} 1$ values and consistent variance across the different MOLLI schemes in humans. This was true even for a wide range of $\mathrm{T} 1$ values with MOLLI schemes with no pause between modified-LookLocker experiments, indicating potential value for myocardial tissue characterization within just 8 heart beats.

The methods presented in this study represent a different approach in quantitative CMR with existing clinical pulse sequences. SQUAREMR allows for correction of quantitative CMR data (e.g. MOLLI T1 maps) that have already been acquired by simulating the clinical pulse sequence that was used for the data acquisition. Last, it is expected that in the future SQUAREMR may improve data consistency and advance quantitative MR to become more robust across imaging centers, vendors and experimental configurations and the technique may be extended in other areas of quantitative MR imaging.

\section{Ethics, consent and permissions}

The study was approved by the local ethics committee and all subjects provided written consent for publication of this study and accompanying images (The regional ethics committee, Lund, Sweden. Ethics application number: 541/2004).

\section{Competing interests}

EH is the founder of Medviso AB, Lund, Sweden, that develops medical image-analysis software. The remaining authors declare that they have no competing interests.

\section{Authors' contributions}

CGX and AHA conceived the study, carried out the experiments and study design and drafted and finalized the manuscript. CGX carried out the SQUAREMR development. SB and GK carried out the development of the phantoms. CGX, SB and GK carried out data collection. EH and HA participated in the design of the study. All authors have contributed to final manuscript and approved it.

\section{Acknowledgements}

Funding was provided by the Greek General Secretariat for Research and Development via an Excellence grant, by the Swedish Heart and Lung Foundation, the Region of Skåne and by the Medical Faculty at Lund University. We would like to thank Mikael Kanski, MD, PhD for his assistance in acquiring the human volunteer images.

\section{Author details}

${ }^{1}$ Cardiac MR Group, Department of Clinical Physiology and Nuclear Medicine, Skåne University Hospital Lund, Lund University, Lund, Sweden. ${ }^{2}$ Department of Computer Science and Biomedical Informatics, University of Thessaly, Lamia, Greece. ${ }^{3}$ Laboratory of Computing and Medical Informatics, School of Medicine, Faculty of Health Sciences, Aristotle University of Thessaloniki, Thessaloniki, Greece. ${ }^{4}$ Department of Biomedical Engineering, Faculty of Engineering, Lund University, Lund, Sweden. ${ }^{5}$ Centre of Mathematical Sciences, Faculty of Engineering, Lund University, Lund, Sweden.

Received: 29 June 2015 Accepted: 15 November 2015

Published online: 26 November 2015

\section{References}

1. Arheden H, Saeed M, Higgins CB, Gao DW, Bremerich J, Wyttenbach R, et al. Measurement of the distribution volume of gadopentetate dimeglumine at echo-planar MR imaging to quantify myocardial infarction: comparison with 99mTc-DTPA autoradiography in rats. Radiology. 1999;211:698-708. 
2. Moon JC, Messroghli DR, Kellman P, Piechnik SK, Robson MD, Ugander M, et al. Myocardial T1 mapping and extracellular volume quantification: a society for cardiovascular magnetic resonance (SCMR) and CMR working group of the european society of cardiology consensus statement J cardiovasc magn reson: off J Soc Cardiovasc Magn Reson. 2013;15:92.

3. Chow K, Flewitt JA, Green JD, Pagano JJ, Friedrich MG, Thompson RB. Saturation recovery single-shot acquisition (SASHA) for myocardial T(1) mapping. Magn Reson Med. 2014;71:2082-95.

4. Fitts M, Breton E, Kholmovski EG, Dosdall DJ, Vijayakumar S, Hong KP, et al. Arrhythmia insensitive rapid cardiac T1 mapping pulse sequence. Magn Reson Med. 2013;70:1274-82.

5. Messroghli DR, Radjenovic A, Kozerke S, Higgins DM, Sivananthan MU, Ridgway JP. Modified Look-Locker inversion recovery (MOLLI) for highresolution T1 mapping of the heart. Magn Reson Med. 2004;52:141-6.

6. Piechnik SK, Ferreira VM, Dall'Armellina E, Cochlin LE, Greiser A, Neubauer S, et al. Shortened modified look-locker inversion recovery (ShMOLLI) for clinical myocardial T1-mapping at 1.5 and $3 \mathrm{~T}$ within a 9 heartbeat breathhold. J cardiovasc magn reson: off J Soc Cardiovasc Magn Reson. 2010;12:69.

7. Weingärtner S, Akçakaya M, Basha T, Kissinger KV, Goddu B, Berg S, et al. Combined saturation/inversion recovery sequences for improved evaluation of scar and diffuse fibrosis in patients with arrhythmia or heart rate variability. Magn Reson Med. 2014;71:1024-34.

8. Cooper MA, Nguyen TD, Spincemaille P, Prince MR, Weinsaft JW, Wang Y. How accurate is MOLLI T1 mapping in vivo? Validation by spin echo methods. PloS one. 2014;9:e107327.

9. Higgins D, Moon J. Review of T1 mapping methods: comparative effectiveness including reproducibility issues. Curr Cardiovasc Imaging Rep. 2014;7:1-10.

10. Kellman P, Hansen MS. T1-mapping in the heart: accuracy and precision. J cardiovasc magn reson: off J Soc Cardiovasc Magn Reson. 2014;16:2.

11. Roujol S, Weingartner S, Foppa M, Chow K, Kawaji K, Ngo LH, et al. Accuracy, precision, and reproducibility of four T1 mapping sequences: a head-to-head comparison of MOLLI, ShMOLLI, SASHA, and SAPPHIRE. Radiology. 2014;272:683-9.

12. Messroghli DR, Greiser A, Frohlich M, Dietz R, Schulz-Menger J. Optimization and validation of a fully-integrated pulse sequence for modified look-locker inversion-recovery (MOLLI) T1 mapping of the heart. JMRI. 2007;26:1081-6.

13. Wacker CM, Bock M, Hartlep AW, Beck G, van Kaick G, Ertl G, et al. Changes in myocardial oxygenation and perfusion under pharmacological stress with dipyridamole: assessment using $T^{*} 2$ and T1 measurements. Magn Reson Med. 1999:41:686-95.

14. Dabir D, Child N, Kalra A, Rogers T, Gebker R, Jabbour A, et al. Reference values for healthy human myocardium using a T1 mapping methodology: results from the International T1 Multicenter cardiovascular magnetic resonance study. J cardiovasc magn reson: off J Soc Cardiovasc Magn Reson. 2014;16:69.

15. Ma D, Gulani V, Seiberlich N, Liu K, Sunshine JL, Duerk JL, et al. Magnetic resonance fingerprinting. Nature. 2013;495:187-92.

16. Gai ND, Stehning C, Nacif M, Bluemke DA. Modified look-locker T1 evaluation using Bloch simulations: human and phantom validation. Magn Reson Med. 2013;69:329-36.

17. Ramanan V, Ghugre NR, Cunningham CH, Stainsby JA, Connelly KA, Leber A, et al. Relaxometry using sequence SimuLation (RUSSL): application to myocardial T1-mapping using MOLLI. Salt Lake City, UT, USA: ISMRM 21th Annual Meeting; 2013.

18. Deichmann R, Haase A. Quantification of T1 values by SNAPSHOT-FLASH NMR imaging. J Magn Reson. 1969;1992(96):608-12.

19. Xanthis $C G$, Venetis IE, Aletras $A H$. High performance MRI simulations of motion on multi-GPU systems. J cardiovasc magn reson: off J Soc Cardiovasc Magn Reson. 2014;16:48

20. Xanthis CG, Venetis IE, Chalkias AV, Aletras AH. MRISIMUL a GPU-based parallel approach to MRI simulations. IEEE Trans Med Imaging. 2014;3:607-17.

21. Wissmann L, Santelli C, Segars WP, Kozerke S. MRXCAT: realistic numerical phantoms for cardiovascular magnetic resonance. J cardiovasc magn reson: off J Soc Cardiovasc Magn Reson. 2014;16:63.

22. Petersson JS, Christoffersson JO, Golman K. MRI simulation using the k-space formalism. Magn Reson Imaging. 1993;11:557-68.

23. Mitchell MD, Kundel HL, Axel L, Joseph PM. Agarose as a tissue equivalent phantom material for NMR imaging. Magn Reson Imaging. 1986;4:263-6.
24. Verhaert D, Thavendiranathan P, Giri S, Mihai G, Rajagopalan S, Simonetti OP, et al. Direct T2 quantification of myocardial edema in acute ischemic injury. J Am Coll Cardiol Img. 2011;4:269-78.

25. Kellman $\mathrm{P}$, Wilson JR, Xue H, Ugander M, Arai AE. Extracellular volume fraction mapping in the myocardium, part 1: evaluation of an automated method. J cardiovasc magn reson: off J Soc Cardiovasc Magn Reson. 2012;14:63.

26. Kellman P, Xue H, Chow K, Spottiswoode BS, Arai AE, Thompson RB. Optimized saturation recovery protocols for T1-mapping in the heart: influence of sampling strategies on precision. J cardiovasc magn reson: off J Soc Cardiovasc Magn Reson. 2014;16:55

27. Schelbert EB, Testa SM, Meier CG, Ceyrolles WJ, Levenson JE, Blair AJ, et al. Myocardial extravascular extracellular volume fraction measurement by gadolinium cardiovascular magnetic resonance in humans: slow infusion versus bolus. J cardiovasc magn reson: off J Soc Cardiovasc Magn Reson 2011;13:16.

28. Ugander M, Oki AJ, Hsu LY, Kellman P, Greiser A, Aletras AH, et al. Extracellular volume imaging by magnetic resonance imaging provides insights into overt and sub-clinical myocardial pathology. Eur Heart J. 2012;33:1268-78

29. Akcakaya M, Basha TA, Weingartner S, Roujol S, Berg S, Nezafat R. Improved quantitative myocardial T2 mapping: Impact of the fitting model. Magn Reson Med. 2015;74(1):93-105.

30. De Graaf RA, Nicolay K. Adiabatic if pulses: Applications to in vivo NMR. Concepts Magn Reson. 1997;9:247-68

31. Heiberg E, Sjogren J, Ugander M, Carlsson M, Engblom H, Arheden H. Design and validation of Segment-freely available software for cardiovascular image analysis. BMC Med Imaging. 2010;10:1

32. Cerqueira MD, Weissman NJ, Dilsizian V, Jacobs AK, Kaul S, Laskey WK, et al. Standardized myocardial segmentation and nomenclature for tomographic imaging of the heart. A statement for healthcare professionals from the cardiac imaging committee of the council on clinical cardiology of the American heart association. Int J Cardiovasc Imaging. 2002;18:539-42.

33. Bland JM, Altman DG. Statistical methods for assessing agreement between two methods of clinical measurement. Lancet. 1986;1:307-10.

34. Kellman P, Herzka DA, Hansen MS. Adiabatic inversion pulses for myocardial T1 mapping. Magn Reson Med. 2014;71:1428-34.

35. Nacif MS, Turkbey EB, Gai N, Nazarian S, van der Geest RJ, Noureldin RA, et al. Myocardial T1 mapping with MRI: comparison of look-locker and MOLLI sequences. J Magn Reson Imaging. 2011;34:1367-73.

36. Henningsson $M$, Botnar $R$, Voigt T. 3D saturation recovery imaging for free breathing myocardial T1 mapping. J Cardiovasc Magn Reson. 2013;15:P44.

37. Robson MD, Piechnik SK, Tunnicliffe EM, Neubauer S. T1 measurements in the human myocardium: the effects of magnetization transfer on the SASHA and MOLLI sequences. Magn Reson Med. 2013;70:664-70.

38. Weber OM, Speier P, Scheffler K, Bieri O. Assessment of magnetization transfer effects in myocardial tissue using balanced steady-state free precession (bSSFP) cine MRI. Magn Reson Med. 2009;62:699-705.

\section{Submit your next manuscript to BioMed Central and we will help you at every step:}

- We accept pre-submission inquiries

- Our selector tool helps you to find the most relevant journal

- We provide round the clock customer support

- Convenient online submission

- Thorough peer review

- Inclusion in PubMed and all major indexing services

- Maximum visibility for your research

Submit your manuscript at www.biomedcentral.com/submit

C) Biomed Central 